\title{
Scaffolded DNA origami: from generalized multi-crossovers to polygonal networks
}

\author{
Paul W.K. Rothemund \\ California Institute of Technology, Pasadena, CA 91125 pwkr@dna.caltech.edu
}

My acquaintance with Ned Seeman began in the Caltech library sometime during 1992. At the time I was trying to design a DNA computer and was collecting papers in an attempt to learn all the biochemical tricks ever performed with DNA. Among the papers was Ned and Junghuei Chen's beautiful construction of a DNA cube [1]. I had no idea how to harness such a marvel for computation - the diagrams explaining the cube were in a visual language that I could not parse and its static structure, once formed, did not seem to allow further information processing. However, I was in awe of the cube and wondered what kind of mad and twisted genius had conjured it.

Ned's DNA sculptures did turn out to have a relationship to computation. In 1994 Len Adleman's creation of a DNA computer [2] showed that linear DNA self-assembly, together with operations such as PCR, could tackle NP-complete computational problems. Excited by this result, Erik Winfree quickly forged an amazing link that showed how the self-assembly of geometrical DNA objects, alone, can perform universal computation [3]. The demonstration and exploration of this link has kept a small gaggle of computer scientists and mathematicians tangled up with Ned and his academic children for the last decade. At an intellectual level the technical achievements of the resulting collaborations and interactions have been significant, among them the first two-dimensional DNA crystals [4] and algorithmic self-assembly of both linear [5] and two-dimensional [6] arrays. By various other paths, a number of physicists have joined the party, mixing their own ideas with Ned's paradigm of "DNA as tinkertoys" to create nanomechanical systems such as DNA tweezers [7] and walkers $[8,9,10]$. DNA nanotechnology has taken on a life of its own since Ned's original vision of DNA fish flying in an extended Escherian lattice [11] and we look forward to a new "DNA world" in which an all-DNA "bacterium" wriggles, reproduces and computes.

On a personal level, I and many others have gotten to find out exactly what kind of twisted genius Ned is. Ned is a singular character. He is at once gruff and caring, vulgar and articulate, stubborn and visionary. Ned is generous both with his knowledge of DNA and his knowledge of life. His 
life's philosophy includes a strong tension between the abysmally negative (the general state of the world) and the just tolerably positive (that which one can, with great effort, hope to achieve). To paraphrase and to whitewash, "In a world full of execrable excresences, there is always a fetid coprostasis of an idea to make your own." Once one is correctly calibrated to Ned, this superficially gloomy counsel becomes positively bright and Ned's success with DNA nanotechnology serves as an example for the young scientist. In fact Ned's education of young scientists reveals a latent optimism. As an advisor Ned plots a strategic course, giving graduate students projects with risks and payoffs calculated to help them succeed at every stage- from confidence builders in their first years to high risk/high gain projects in later years.

Ned's own relationship with science is equally telling of his character. He is healthily (and vocally) paranoid about Nature's determination to screw up his experiments. To combat this he practices a capricious paganism, frequently switching between gods in the hope that one will answer his prayers for a highly-ordered three-dimensional DNA crystal. (A habit which he attempted to break unsuccessfully when he abandoned crystallography.) Such superstition is tongue in cheek however, and Ned is one of the most careful scientists that I know. He is ever-mindful that, as Peter Medawar wrote, "research is surely the art of the soluble" and while his highly imaginative research is constructive and non-reductionist in its goals, Ned makes sure that it rests on falsifiable Popperian bedrock.

In celebration of Ned the character, as well as the box of tinkertoys and legos that he has created, I cover two topics. First, I review the recent generalization of Ned's geometry of parallel crossovers to the creation of arbitrary shapes and patterns via a method called scaffolded DNA origami. I give an example pattern with roughly 200 pixels spaced 6 nanometers apart. Second I propose a new method for using scaffolded DNA origami to make arbitrary polygonal networks, both two-dimensional planar stick-figures and three dimensional polyhedra.

\section{Scaffolded DNA origami for parallel multicrossovers}

Fig. 1a and $\mathrm{b}$ show one of the most successful of Ned's noncanonical DNA motifs, a 'double-crossover' molecule [12] fashioned from two parallel double helical domains that comprise four distinct strands of DNA. Each DNA strand winds along one helix for a number of bases before switching to the other helix by passing through a structure called a 'crossover' (small black triangles). Because strands reverse direction at the crossovers, the crossovers are termed 'antiparallel'. It is the juxtaposition of two crossovers that holds the helices in their parallel arrangement (isolated crossovers assume an equilibrium angle of roughly 60 degrees), and it is their juxtaposition that holds the helices rigidly together (isolated crossovers are floppy). These properties allow double crossovers to assemble into large extended lattices [4], and nanotubes [13]. 
a

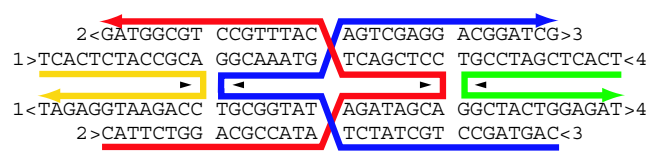

C

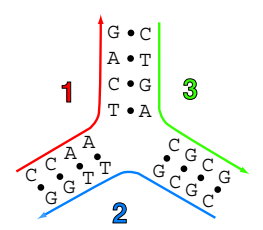

multi-stranded

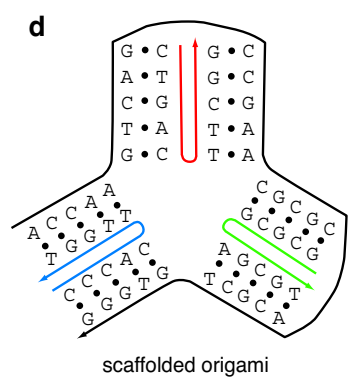

b

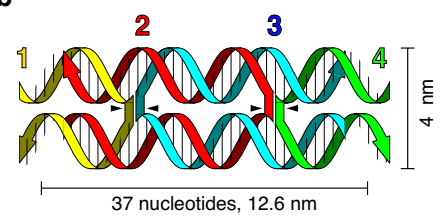

e

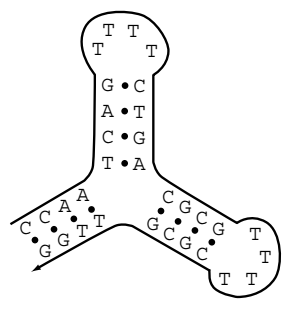

single-stranded origami

Fig. 1. Double-crossover molecules and flavors of DNA design.

The idea of holding helical domains in a parallel arrangement via the juxtaposition of antiparallel crossovers has become a general principle in DNA nanotechnology, used in at least a dozen constructions. For example, it has been extended to molecules with three parallel helixes [14] and it has been used to attach triangles rigidly to a nanomechanical device [15].

A key question is how to create generalized multi-crossover molecules with parallel helices. To answer this question, it is necessary to understand the advantages and disadvantages of different approaches. Within the DNA nanotechnology paradigm, designs may be classified by how they are built up from component strands, being (1) composed entirely of short oligonucleotide strands as in Fig. 1c, (2) composed of one long 'scaffold strand' (black) and numerous short 'helper strands' (colored) as in Fig. 1d, or (3) composed of one long strand and few or no helpers as in Fig. 1e. Here these design approaches are termed 'multi-stranded', 'scaffolded', and 'single-stranded', respectively. The last two are termed 'DNA origami' because a single long strand is folded, whether by many helpers or by self-interactions.

Multi-stranded designs (such as Ned's original cube) suffer from the difficulty of getting the ratios of the component short strands exactly equal. If there is not an equal proportion of the various component strands then incomplete structures form and purification may be required. Because, for large and complex designs, a structure missing one strand is not very different from a complete structure, purification can be difficult and may have to be performed in multiple steps. Single-stranded origami such as William Shih's octahedron [16] cannot, by definition, suffer from this problem. Scaffolded origami sidesteps the problem of equalizing strand ratios by allowing an excess of helpers to be used. As long as each scaffold strand gets one of each 


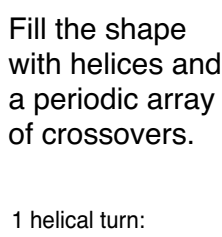

Fill the shape a periodic array of crossovers.

1 helical turn: with helices and

c Add helper strands to bind the scaffold together.
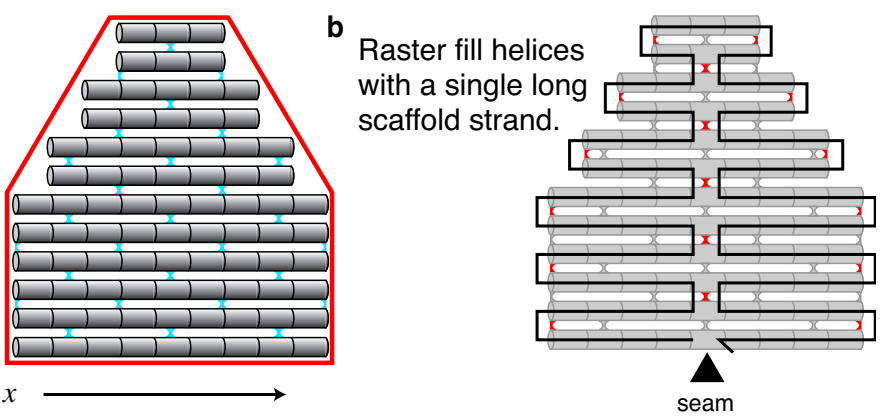

Special helper strands $(\diamond)$ bridge the seam.

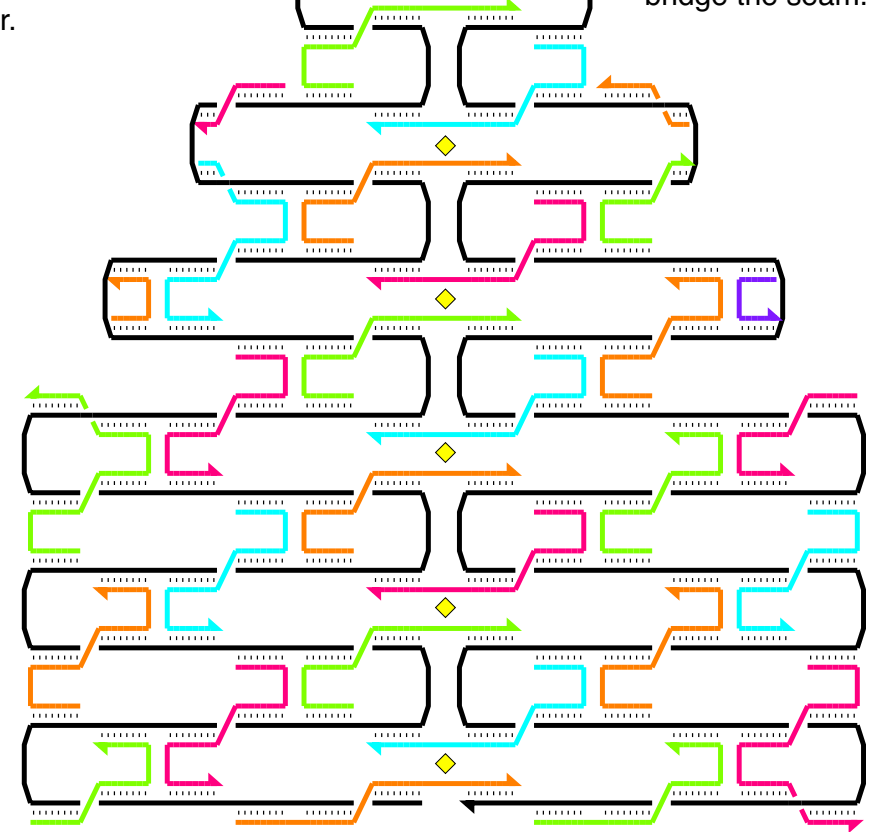

Fig. 2. Design of DNA origami.

helper, all scaffolds may fold correctly (some might get trapped in misfoldings). Because origami are easily differentiable from the helpers, separating them is not difficult (e.g. large origami stick much more strongly to mica surfaces than do tiny helpers and so excess helpers can be washed away).

Single-stranded origami and scaffolded origami thus seem the best candidates for the creation of large complex structures. As Shih has observed [personal communication], the geometry used for the octahedron should generalize and allow the creation of arbitrary polygonal networks. However, the 
use of single-stranded origami to create parallel multi-crossover designs seems difficult (but perhaps only to me).

Generalization of the parallel helical geometry introduced by doublecrossover molecules is simple using scaffolded DNA origami; I have recently demonstrated the technique for the creation of six arbitrary shapes and six arbitrary patterns (including the one shown here); the design method and experiments showing its generality are described in reference [17]. To get a feeling for the method, look at Fig. 2. Shapes are approximated by laying down a series of parallel helical domains inside of the shape (Fig. 2a). Helices are cut to fit the shape, in a series of sequential pairs from top to bottom, so that the resulting geometry approximates the shape within one DNA turn $(\sim 3.6 \mathrm{~nm})$ in the $x$-direction and two helical widths $(\sim 6 \mathrm{~nm}$, including an inter-helix gap) in the $y$-direction. To make a molecular design, a scaffold is run exactly once through each helix; performed in a raster-fill manner, this creates a 'folding path' (Fig. 2b). To hold the scaffold in this shape, helper strands are added to create a regular pattern of antiparallel crossovers (Fig. 2c).

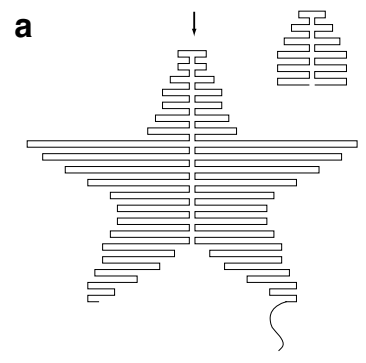

b
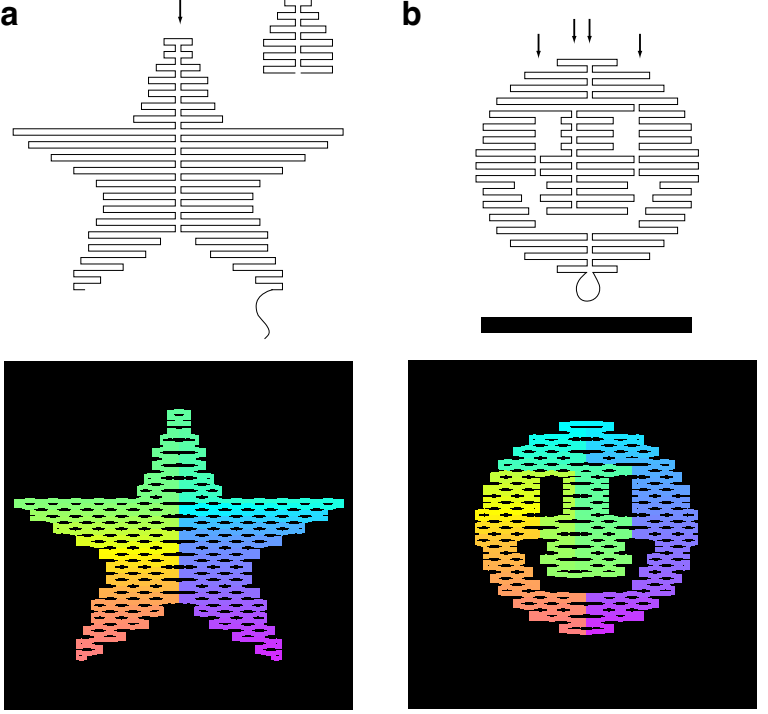
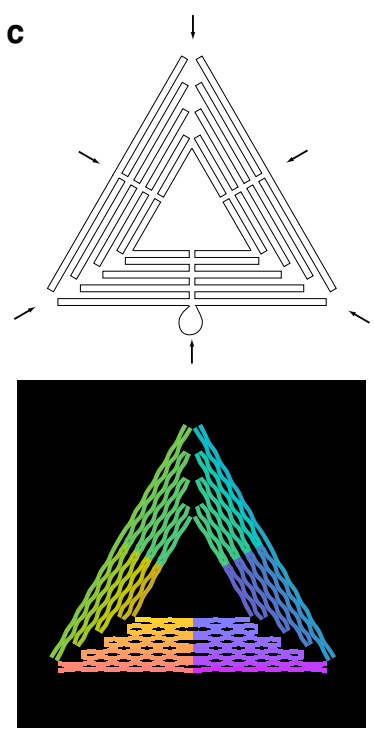

Fig. 3. Several folding paths (top) drawn without helper strands and predicted structures (bottom) that use a 7000-base-long scaffold. Colors indicate the base position on the scaffold from 1 (red-orange) to 7000 (purple). Arrows indicate seams which are bridged by helper strands for mechanical stability. Scale bar, $100 \mathrm{~nm}$.

As reported in [17], the method is general and scales quite well to large origami (Fig. 3). The two shapes diagrammed in Fig. 3b and c each form in excess of $70 \%$ yield, and each uses a 7000-base-long scaffold requiring more than 200 DNA strands for a final molecular weight of 15,000 nucleotides. Thus 
these DNA origami have a molecular weight $100 \times$ that of the original doublecrossover and almost $6 \times$ larger than Ned's largest geometric construction, a truncated octahedron [18]. Further, such scaffolded origami are created in a single laboratory step: strands are mixed together in a $\mathrm{Mg}^{2+}$-containing buffer and annealed from $90^{\circ} \mathrm{C}$ to $20^{\circ} \mathrm{C}$ over the course of 2 hours.

Given a shape, such as the rectangle in Fig. $4 \mathrm{a}$ and b, it is simple to decorate it with an arbitrary pattern of binary pixels. The position of each helper strand (of which there are roughly 200) is considered to be a pixel. The original set of helper strands is taken to represent binary ' 0 's. To represent binary '1's a new set of labelled helper strands is constructed; so far they are labelled with extra DNA hairpins. To create a desired pattern (say Fig. 4c) the appropriate complementary sets of strands are drawn from the original helper strands and the labelled helper strands. Everywhere the pattern has a ' 0 ' an original helper strand is used, everywhere the the pattern has a ' 1 ' a new helper strand is used. Creating the mixture of strands for a desired pattern requires about 1.5 hours of pipetting.

a

b

C
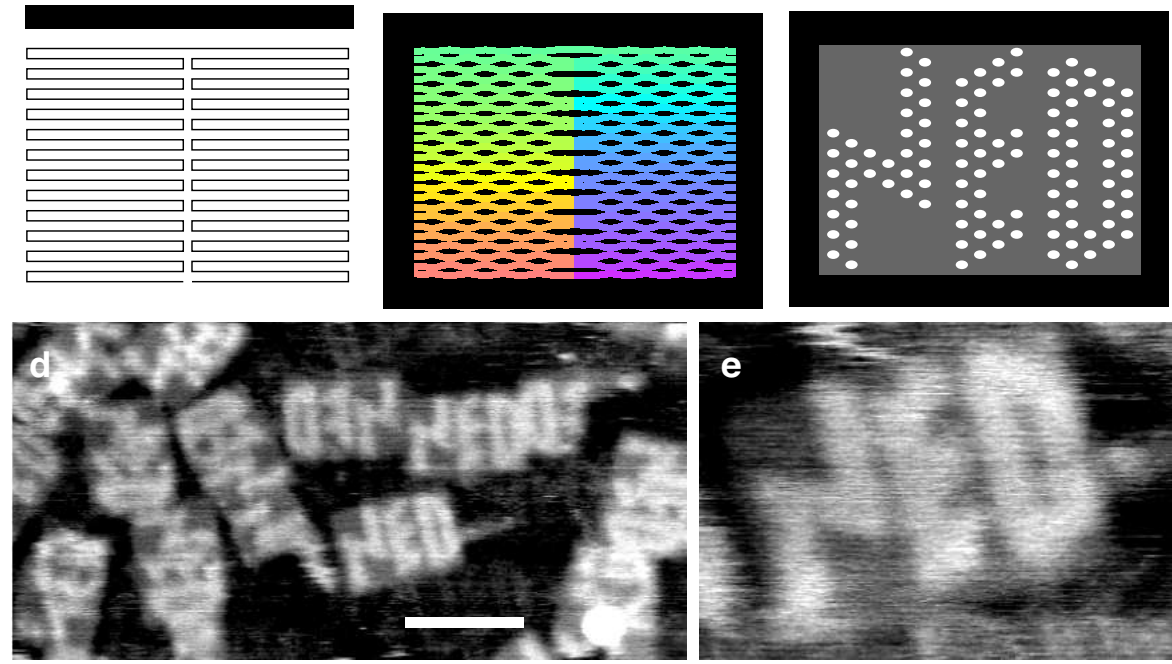

Fig. 4. An arbitrary pattern. White features are DNA hairpins. The black scale bar in $\mathbf{a}$ applies to $\mathbf{b}, \mathbf{c}$ and $\mathbf{e}$ as well. Both black and white scale bars, $100 \mathrm{~nm}$.

The pattern in Fig. 4c was made in this manner, just for this paper. Fig. 4d and e show atomic force micrographs of the result; hairpin labels appear as light dots, unlabelled positions appear gray, and the mica surface on which the sample is deposited appears black. Each letter is approximately $60 \mathrm{~nm}$ tall (letters half this height are shown in ref. [17]). Roughly 50 billion copies of the pattern were made; copies stick to eachother along their vertical edges 
via blunt-end stacking. Note that the pattern clearly shows the influence of Ned on DNA nanotechnology.

Because scaffolded DNA origami makes the the creation of arbitrary shapes and patterns so simple, and because it provides the ability to pattern at the $6 \mathrm{~nm}$ length scale, scaffolded origami has the potential to play a important role in future lithographic techniques for nanocircuits and other nanodevices.

\section{DNA origami for polygonal networks}

Given the ease with which scaffolded origami generalizes parallel crossovers, a question becomes, what other general methods of creating shapes might there be? The first thing that would probably spring to a geometer's mind is the use of polygons. Indeed the attempt to create polygonal networks - DNA stick figures - was where Ned began his quest for 3D structure[11, 19]. His original vision was to "trash the symmetry" of DNA branch junctions to create immobile motifs which could then be assembled into polygonal networks via sticky ends (Fig. 5a and b). Unfortunately, it wasn't that easy; single branched junctions resisted crystallization into 2D lattices for many years. In general, branched junctions formed from single helices are floppy and tend to cyclize into families of trimers, tetramers, and higher macrocycles. In particular, fourarmed branch junctions vacillate between one of two different "stacked-X" conformations [20,21] and, demonstrating a mind of their own, assume a 60 degree angle rather than the 90 degree angle one might like them too. Again by trashing symmetries, one can use specific sticky ends that force a particular connectivity, such as the DNA cube [1], but because of uncertainty in the junction geometry, it is still unknown whether the DNA cube was cube or some other parallelopiped.

It was out of such frustrations that the parallel helical geometry used by Ned to create the double-crossovers was born [12], giving us DNA "lego" bricks rather than the "tinkertoy" spools and sticks originally envisioned. DNA lattices from unconstrained 4-arm junctions were eventually formed either by letting the junctions have their way, to create rhomboidal lattices with 60 degree angles [22], or incorporating symmetries that apparently force the junctions to crystallize into lattices of parallel helices [23]. None of these experiments, however, gets us any closer to tinkertoys.

Recently, in an attempt to create DNA motifs with a square 1:1 aspect ratio, Hao Yan and Thom LaBean came up with what they call a " $4 \mathrm{x} 4$ " motif (Fig. 5c). By using two DNA helices rather than one for each arm of their 4 -arm motif, and connecting these arms with apparently floppy junctions, they have created a motif that crystallizes into rectilinear domains several microns in size [24]. Chengde Mao has modified the $4 \times 4$ to create 3 -arm motifs (Fig. 5d), which he calls "3-point stars", that crystallize beautifully into 30-micron hexagonal lattices [25]. It is amazing that the combination of single covalent bonds and poly-T linkers at the centers of these motifs yield 


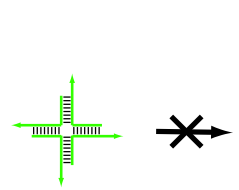

c
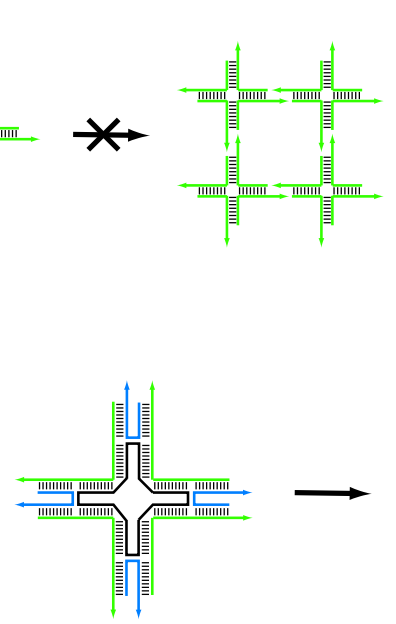

b
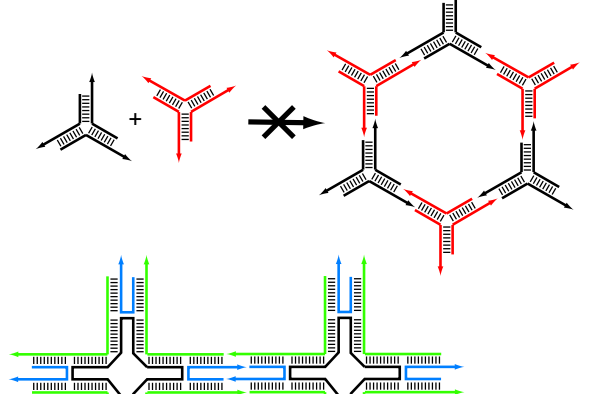

d
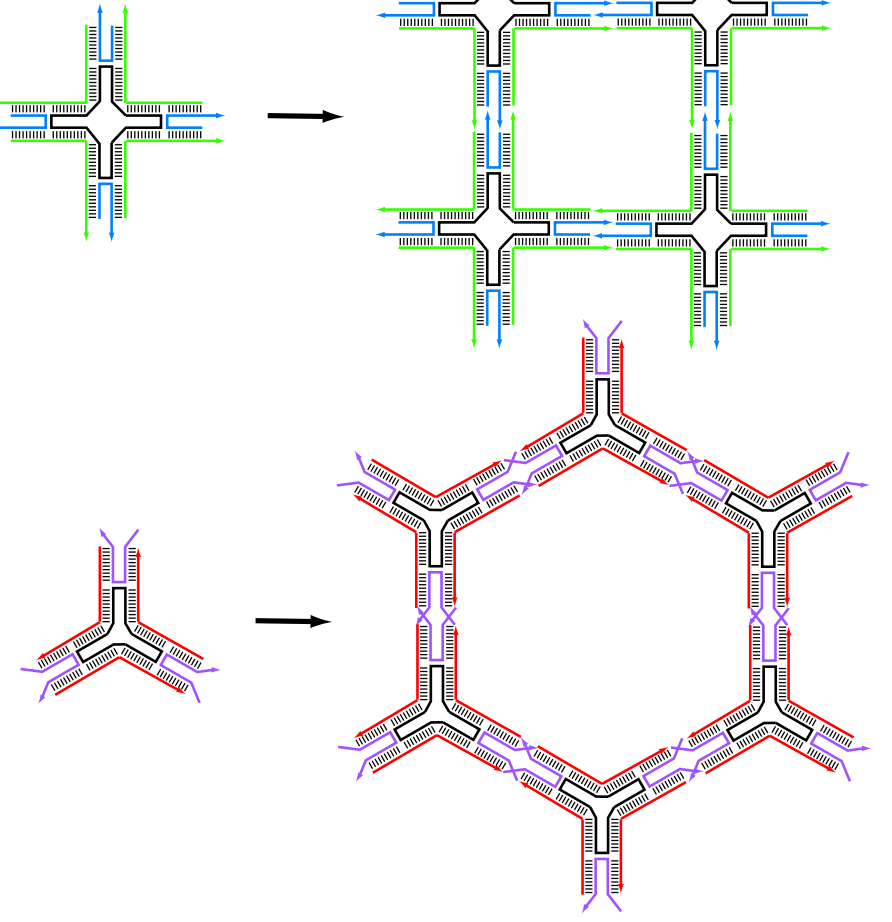

Fig. 5. Ned's original vision for branch junction lattices and the motifs that have succeeded them. Sticky end placement and arm lengths in $\mathbf{c}$ and $\mathbf{d}$ are not accurate; refer to refs. [24] and [25] for actual structures.

structures rigid enough to form large lattices. These successes hint that the principle may be generalized to other numbers of arms - and may provide us with the sticks and spools for DNA tinkertoys.

Here I propose a new multi-arm motif, similar to the $4 \mathrm{x} 4 \mathrm{~s}$ and 3 -point stars in that it uses two helical domains per arm, that may be used in the context of scaffolded DNA origami to create arbitrary polygonal networks. I begin by describing its use to create arbitrary pseudohexagonal networks. 
a

b<smiles>CC(C)C(C)C(C)C(C)C(C)C</smiles>
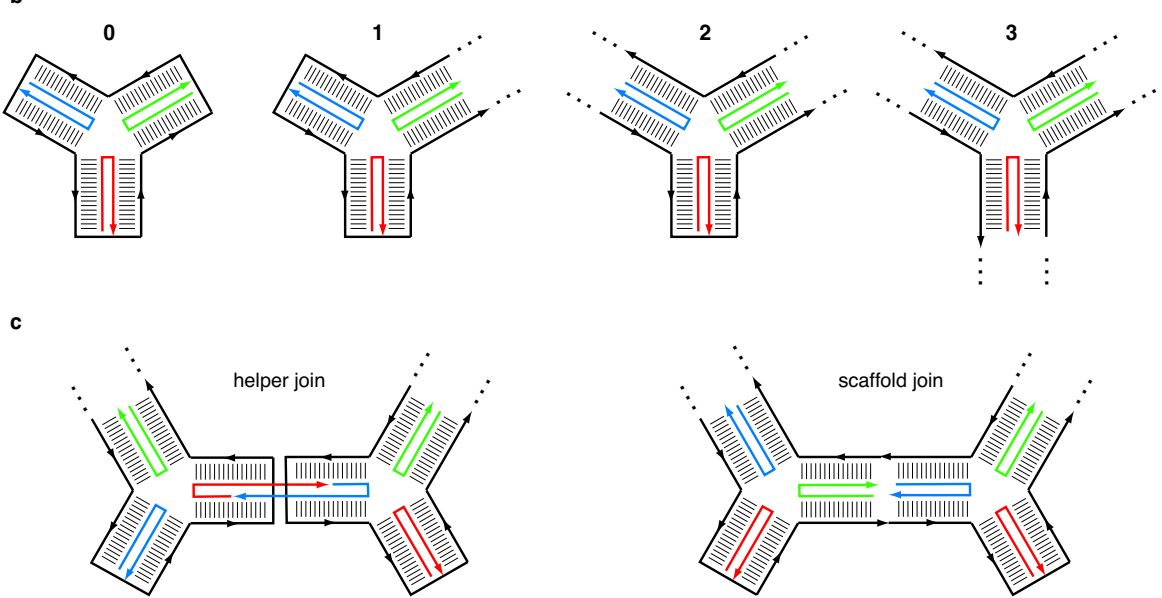

d
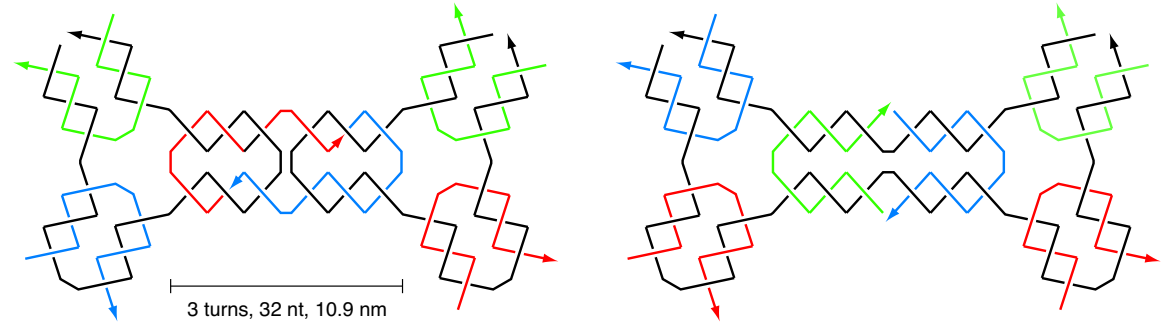

Fig. 6. A pseudohexagonal network composed of geometrical 3-stars and the DNA 3 -stars used to build a molecular approximation.

Fig. 6a shows what is meant by pseudohexagonal networks: planar figures composed from the two 3 -armed components at left (here called 3-stars) without rotation or bending. I propose that such structures can be created from scaffolded DNA origami by replacing each geometrical 3-star with one of the DNA 3-stars diagrammed in Fig. 6b. ${ }^{1}$ In each DNA 3-star, the black strand is intended to be the scaffold strand of a DNA origami and the colored strands are helper strands, each 32 nucleotides long. DNA 3-stars are classified by the

\footnotetext{
${ }^{1}$ Technically, this motif should be called a 1.5-turn DNA 3-star; any odd number of half-turns may be used in the arm.
} 
number of 'open ends' that they have, i.e. the number of breaks in the scaffold strand as it travels around the circumference of the DNA 3-star. Thus DNA 3 -stars can be of 'type-0', 'type-1', 'type-2', or 'type-3'. The type-0 DNA 3star is the simplest pseudohexagonal network; each arm is closed at the end by the scaffold as it crosses from one helix of the arm to the other. Note that these DNA 3-stars differ from Mao's 3-point stars (as well as the 4x4s) in that they have crossovers at the junction between arms, rather than in the middle of each arm - thus it is uncertain how DNA 3-stars will behave in the laboratory. Let's assume for now that they will form well.

When two DNA 3-stars abut in a pseudohexagonal network, they can be joined in one of two ways: either two closed ends meet (Fig. 6c, left) or two open ends meet (Fig. 6c,right). If two closed ends meet then they are mechanically joined by modified helper strands that cross the ends closed by the scaffold strand; call this structure a 'helper join'. ${ }^{2}$ On the other hand, if two open ends meet then they are joined by the scaffold strand - the scaffold strand passes along the top helix from right to left, and returns along the bottom helix from left to right. I call this structure a 'scaffold join'. Fig. 6d shows the helical representation of both helper and scaffold joins.

Given an arbitrary pseudohexagonal network of $N 3$-stars, a simple algorithm allows a molecular design $M$ to be built up from $N$ DNA 3-stars. Fig. 7a shows an example network; Fig. 7b shows simplified diagrams of DNA 3 -stars that show only the scaffold strand and are colored according to their type. The algorithm begins by placing a type-0 DNA 3 -star over a randomly chosen 3-star in the network; Fig. 7c and d show one particular choice, Fig. 7e shows another. The algorithm proceeds by adding type-1 DNA 3-stars one at a time, until the entire network is covered (Fig. 7c,d and e, step 2 through step 7). Each time a type-1 DNA 3-star is added, it is positioned next to an already-placed DNA 3-star (which such position may be chosen randomly) and it is fastened to the already-placed DNA 3-star by a scaffold join. Thus the type of the already-placed 3-star is incremented by 1 (visualized in Fig. 7 as a color change). If the type-1 DNA 3-star is placed next to two or more already-placed DNA 3-stars (Fig. 7d and e, step 7), then it is fastened to one of the DNA 3-stars (chosen randomly) by a scaffold join and to the remaining DNA 3-stars by helper joins (arrows, Fig. 7c, d, and e). Before each addition of a type-1 DNA star, the scaffold is a single closed loop. At the end of each addition, the scaffold is still a single closed loop. Thus the algorithm always generates a design $M$ that has a single continuous scaffold strand.

As described the algorithm is non-deterministic and can generate different folding paths; the position of helper and scaffold joins in $M$ depends on the order in which 3-stars are replaced by DNA 3-stars. ${ }^{3}$ In small designs, such

\footnotetext{
${ }^{2}$ Here each helper strand is drawn as binding to 24 bases in one DNA 3-star, and 8 bases in the other. This is by analogy with similar joints in previously created scaffolded origami; what lengths may work the best are unknown.

${ }^{3}$ Note that the number of scaffold and helper joins in $M$ remains the same, independent of the order in which $M$ is built. By construction, the number of scaffold
} 
a

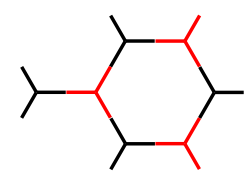

1

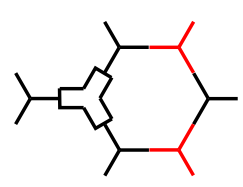

2

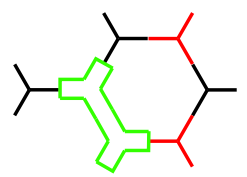

3

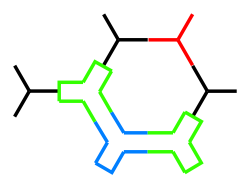

4

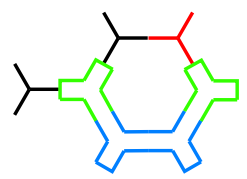

5

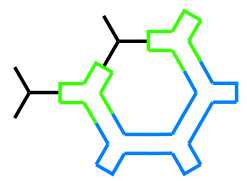

6
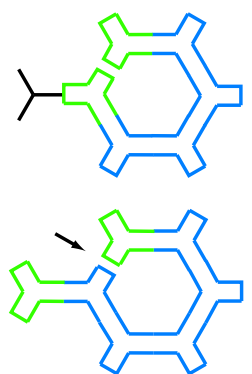
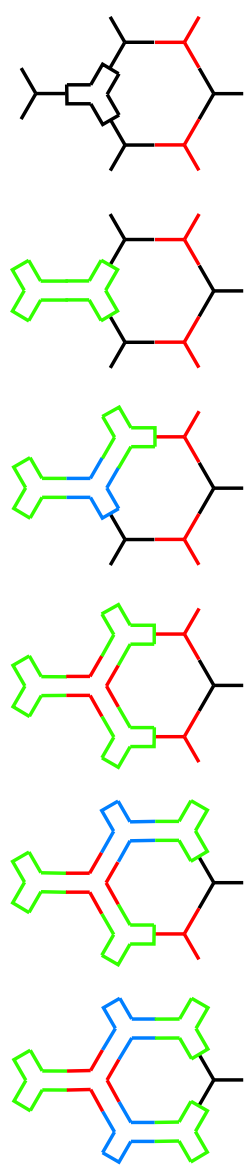

b

एँ ${ }^{0} v^{2} \quad{ }^{3}$

e
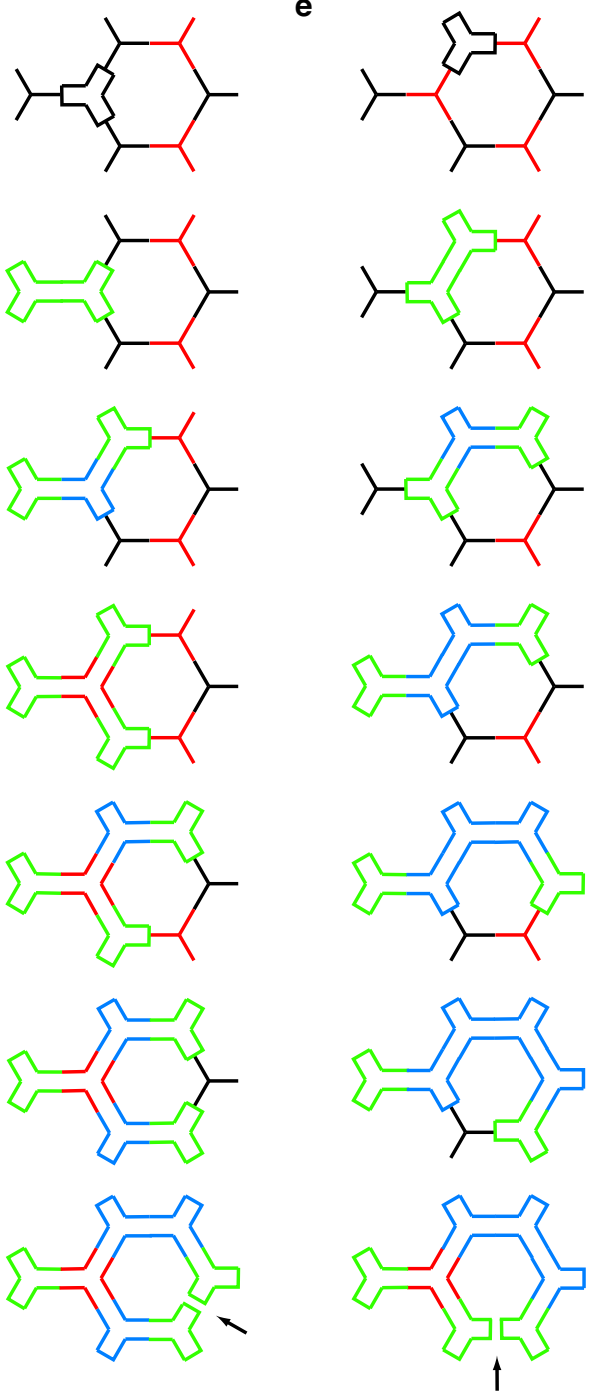

Fig. 7. A pseudohexagonal network, converted to a molecular design in three different ways. Arrows point to helper joins. 
as in Fig. 7, the pattern of scaffold and helper joins seems irrelevant. In large designs, such as those in Fig. 8, it is easy to imagine that the pattern of joins may have bearing on whether the structures fold correctly or on their mechanical stability. For example, perhaps local folds form faster than long distance ones causing short wiggly paths to fold more reliably than long straight ones; if true then the tree-like folding path of the design in Fig. 8c might fold more robustly into a triangular figure (Fig. 8a) than the comb-like folding path of the design in Fig. 8b. Or we might expect that the folding path of Fig. 8e (for which every radius of the hexagon intersects at least two covalent scaffold bonds) will yield a more mechanically stable version of Fig. 8d than the folding path of Fig. $8 \mathrm{f}$ (for which one radius of the hexagon - the dotted line - intersects only helper joins). If it is learned that the pattern of scaffold and helper joins matters, such information can be incorporated into the design algorithm.

Technically, large designs such as those in Fig. 8 seem within easy reach (at least to try). The triangular network (Fig. 8a) would require a 5856-base-long scaffold and the hexagonal ring (Fig. 8b) a scaffold 6912 bases long (rendered using 1.5-turn DNA 3-stars).

While polygonal networks are planar graphs, the objects created with them need not be planar. Fig. 9 (top left) reproduces Ned's proposal for a singlestranded dodecahedron, drawn twisting around the Schlegel diagram ${ }^{4}$ for a dodecahedron. In this scheme the single blue strand that winds around the dodecahedron must leave the dodecahedron once per face, and jump to an adjacent face (Fig. 9, bottom right, makes this path clear). Ned's plan was to cut off these exocyclic arms with restriction endonucleases after the dodecahedron had folded. More inconvenient than the surplus arms is that this structure is a formal knot - in order for it to fold the single strand would have to be cut (say at the black arrow) and threaded through itself many times (at least twice per edge as drawn).

joins, $S$, equals $N-1$ where $N$ is the number of 3 -stars. The number of helper joins, $H$, is obviously $J-S$ where $J$ is the total number of joins (determined by the network geometry). More fun (and perhaps useful) than counting $J$ or $H$ is to observe that $H$ is the number of 'holes' in the network. If the network is embedded in the plane, the number of holes is the number of unconnected regions that the network divides the plane into, disregarding the region outside of the network. For example, the network in Fig. 8a has 21 holes (small hexagons) and the molecular designs Fig. 8b and c both have 21 helper joins. The network in Fig. 8d has 19 holes (18 small hexagons and 1 large interior hexagonal void) and designs Fig. 8e and $\mathrm{f}$ both have 19 helper joins. The relationship $J=S+H=N-1+H$, is just a restatement of Euler's theorem for planar graphs $V-E+F=2$ where the number of vertices $V=N$, the number of edges $E=J$, and the number of faces $F=H+1$ (the number of faces of a graph includes all the holes, plus the region of the plane outside the graph.)

${ }^{4}$ A Schlegel diagram for a polyhedron is just the planar graph associated with that polyhedron. 
a

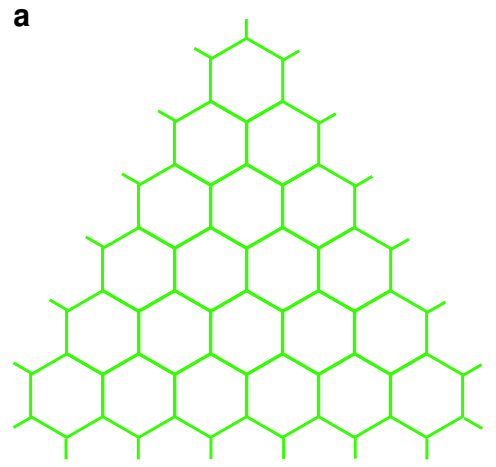

b

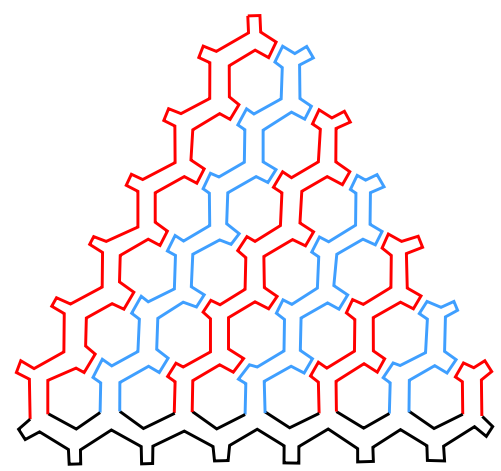

c

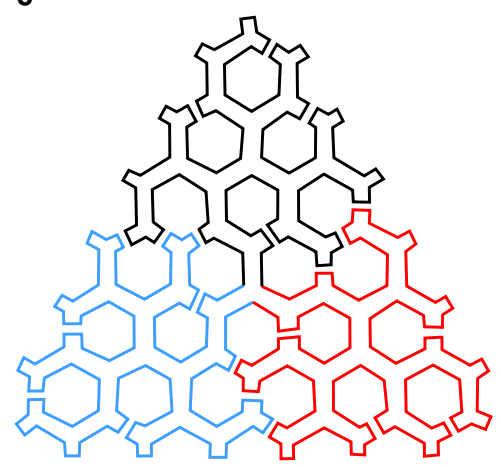

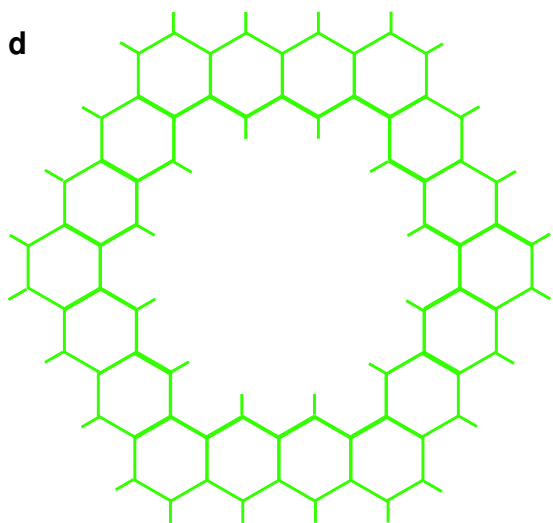

e

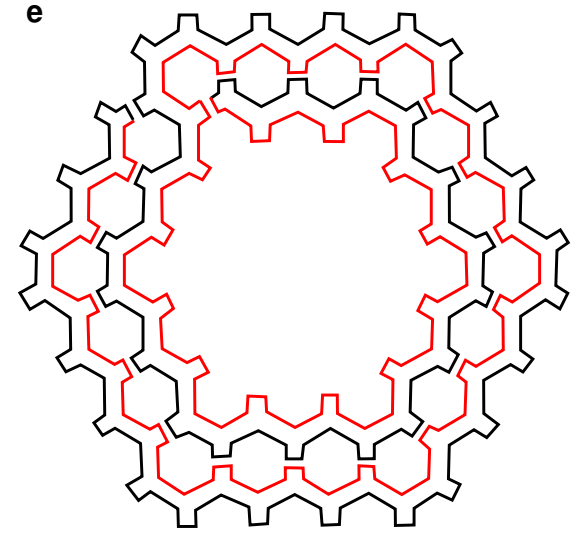

f

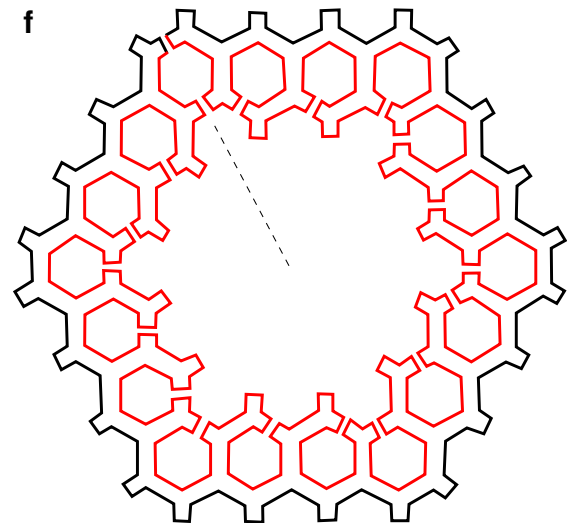

Fig. 8. Given a particular network, folding paths in molecular designs are not unique. Vertically oriented scale bar, $100 \mathrm{~nm}$. 


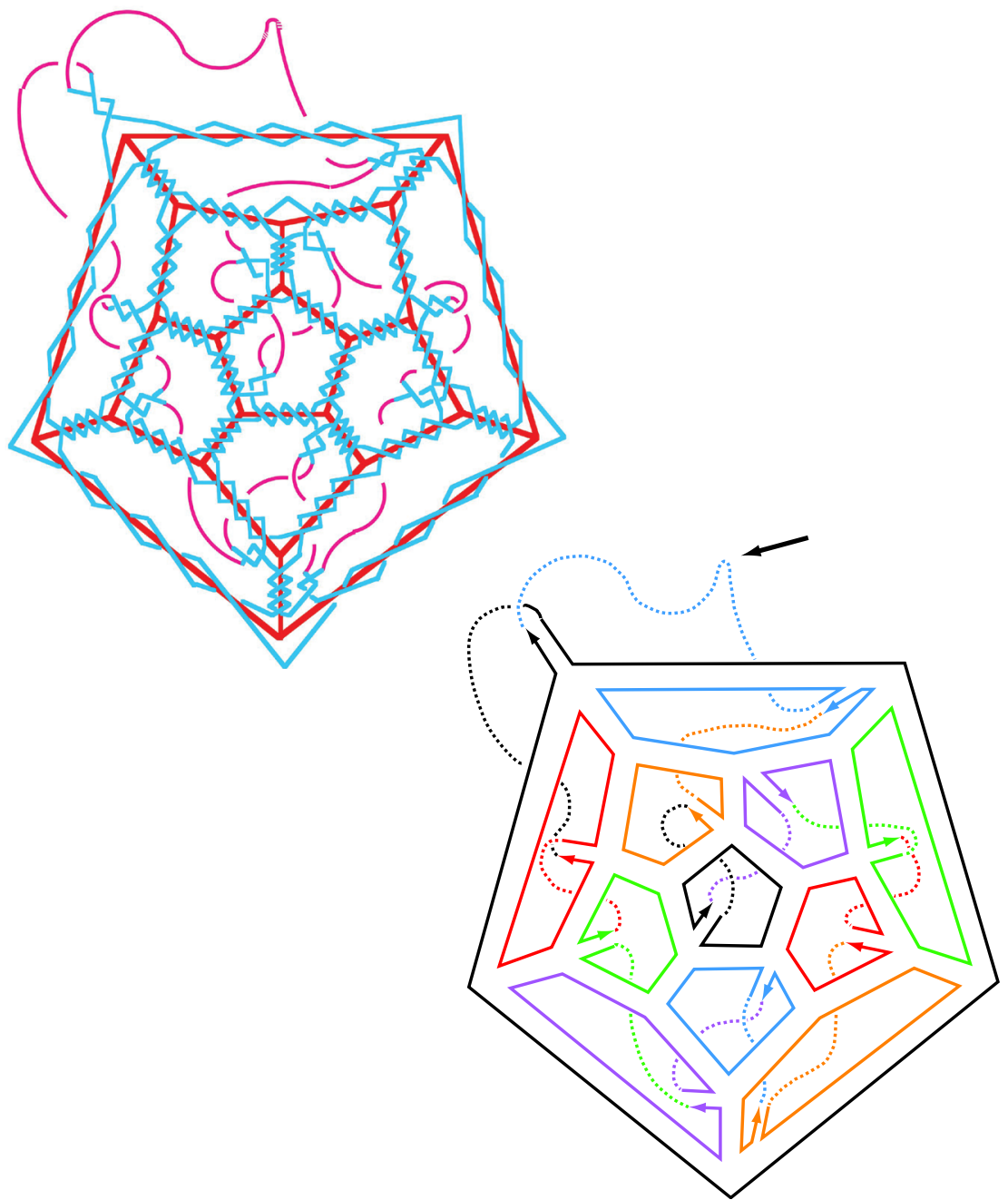

Fig. 9. Ned's vision of a single-stranded dodecahedron. (top left, figure credit: Ned Seeman) Eleven faces of the dodecahedron are represented as interior pentagons of the Schlegel diagram; the twelfth face is the pentagon formed by the outer edges.

If DNA 3-stars tolerate angles other than 120 degrees, a scaffolded origami approach (Fig 10a and b) would allow the dodecahedron to be created without any knotting of the scaffold strand ${ }^{5}$. As designed the folding path visits each vertex in a spiral pattern, spiralling out 5' to 3' from the center along the red contour and spiralling back in along the black counter. More tree-like folding paths similar to that of Fig. 8, bottom left, are obviously possible but

${ }^{5}$ Shih's single-stranded approach would also eliminates such knots. 
it is my intuition that a spiral folding path will leave the smallest possibility of misfoldings. ${ }^{6}$ The dodecahedron uses only 12 DNA 3-stars - using the standard 7000-base scaffold would thus allow the use of larger DNA 3-stars with longer arm lengths (and requiring more than two helper strands per arm). Using 5.5-turn DNA 3-stars, edge lengths would be 11 turns (116 bases) and the total scaffold would be 6960 bases long. Each edge would be $39.4 \mathrm{~nm}$ and the diameter of a sphere enclosing the dodecahedron would be $110 \mathrm{~nm}$.

Ned has described his work on geometrical DNA constructs as "pure Buckminster Fuller". Scaffolded origami may now allow the simple construction of a "DNA buckyball" (Fig 10c and d show the Schlegel diagram and molecular design), a DNA analog of the carbon allotrope fullerene or $\mathrm{C}_{60}$. Using 1.5turn DNA 3-stars, such an analog would only require a 5760 base scaffold and would thus be a little smaller and less complex than current scaffolded designs. Carbon buckyballs are $.7 \mathrm{~nm}$ in diameter - a DNA buckyball would be $50 \mathrm{~nm}$ in diameter and have over $300,000 \times$ the volume. Probably too floppy to image well with atomic force microscopy, DNA buckyballs (and dodecahedra) would have to be characterized by an electron microscopy technique such as single particle analysis or electron tomography.

While I have so far presented structures created from DNA 3-stars, it is possible that scaffolded polygonal origami can be created from other $k$ stars (Fig. 11). DNA 4-stars seem likely to be well-behaved because the $4 \times 4$ molecules are so well-behaved. DNA 5 -stars tolerant of the appropriate angles would make scaffolded icosahedra possible (5.5-turn DNA 5-stars would yield icosohedra with a $75 \mathrm{~nm}$ enclosing sphere and 6960-base scaffold). Eventually, as $k$ increases, a star's central section is likely to become so floppy that it collapses and admits blunt-ended stacking between pairs of helices in opposing arms. My intuition is that this is the major obstacle to high $k$-stars rendered in DNA. Figures of stars of mixed valence may also be possible. Note that the algorithm for constructing a molecular design (adding type-1 stars) is the same for $k>3$ and mixed valence designs; also the number of scaffold joins remains $N-1$ and, because the polygonal networks considered here are all planar graphs, the number of helper joins remains the number of holes.

It will be interesting to see whether polygonal origami works as well as parallel multicrossover origami in the lab - if so it will be a another example of a system for creating a general class of DNA shapes. With a wealth of structural experience under its belt, the DNA nanotechnology community is exploring such generalized approaches for a variety of motifs. For example, William Sherman has proposed a neat framework [26] for the creation of DNA nanotubes of arbitrary cross section. In another example, as discussed

\footnotetext{
${ }^{6}$ This intuition is in opposition to my previous suggestion for why tree-like folding paths might fold better. My imagination is that the more long branches there are floating about, the higher the probability of unintended catenation, for example that two faces of a polyhedron might form in an interlocking manner. Lots of imaginations are possible. I hope that someday some new technique will allow us to make movies of the process and give us a real intuition for folding.
} 

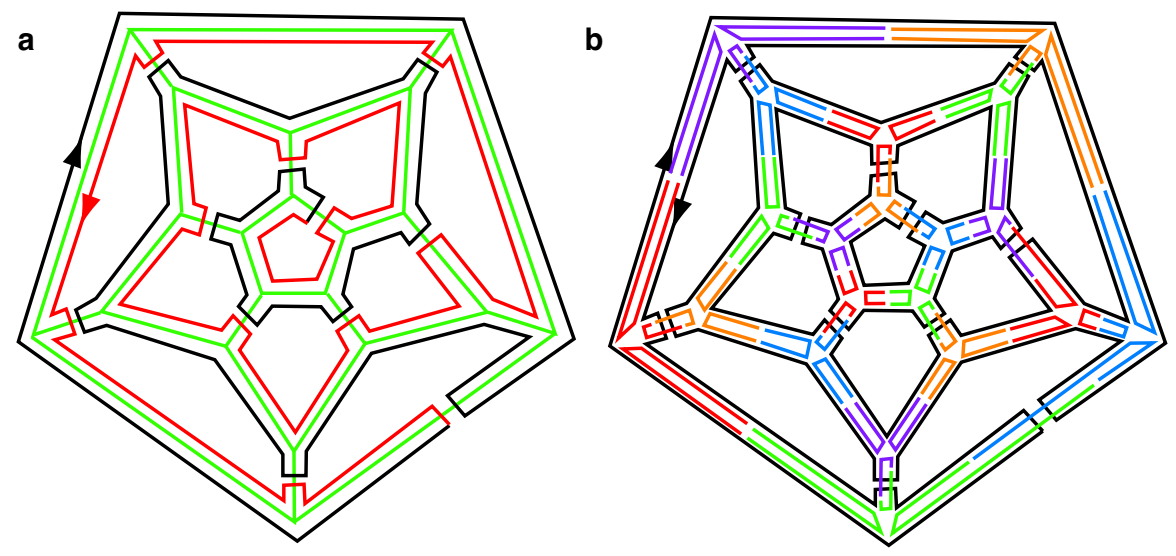

C

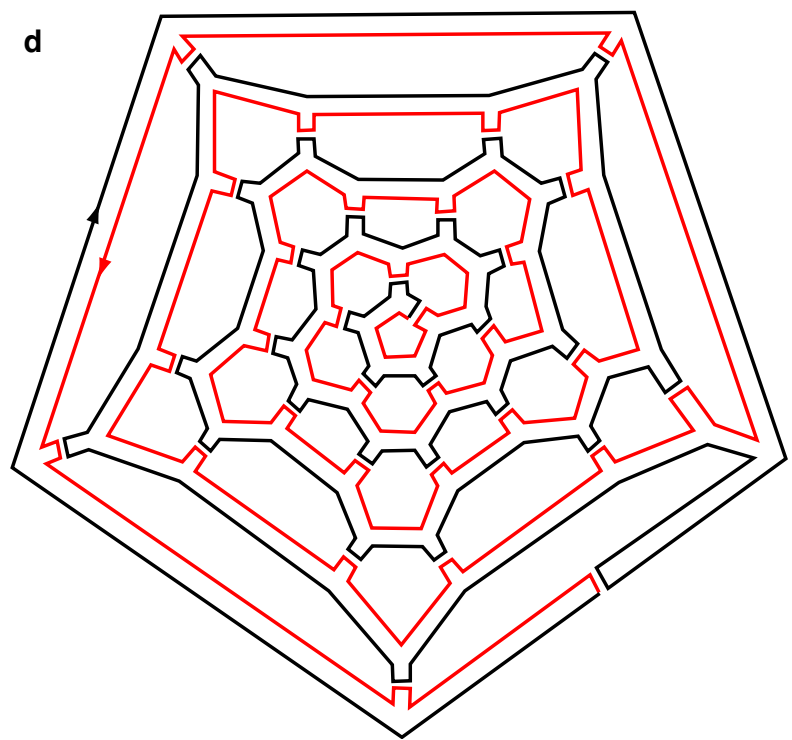

Fig. 10. A dodecahedron and buckyball designed as scaffolded origami. DNA 3stars are asymmetric and have a distinct 'top' and 'bottom' face. It is unclear if this will result in one or two forms (each inside-out of the other) for each polyhedron.

above, William Shih has observed that single-stranded origami may be used to create arbitrary polygonal networks. ${ }^{7}$ Ideally, for every motif that we create, we would have such a scheme for composing the motif into larger arbitrary structures. In this, some motifs present stimulating and difficult challenges. Ned's surprising paranemic crossover DNA [27] might be generalized to form

\footnotetext{
7 To see this, replace helper joins with paranemic cohesion motifs and scaffold joins with Shih's double-crossover struts in all the diagrams of this section.
} 


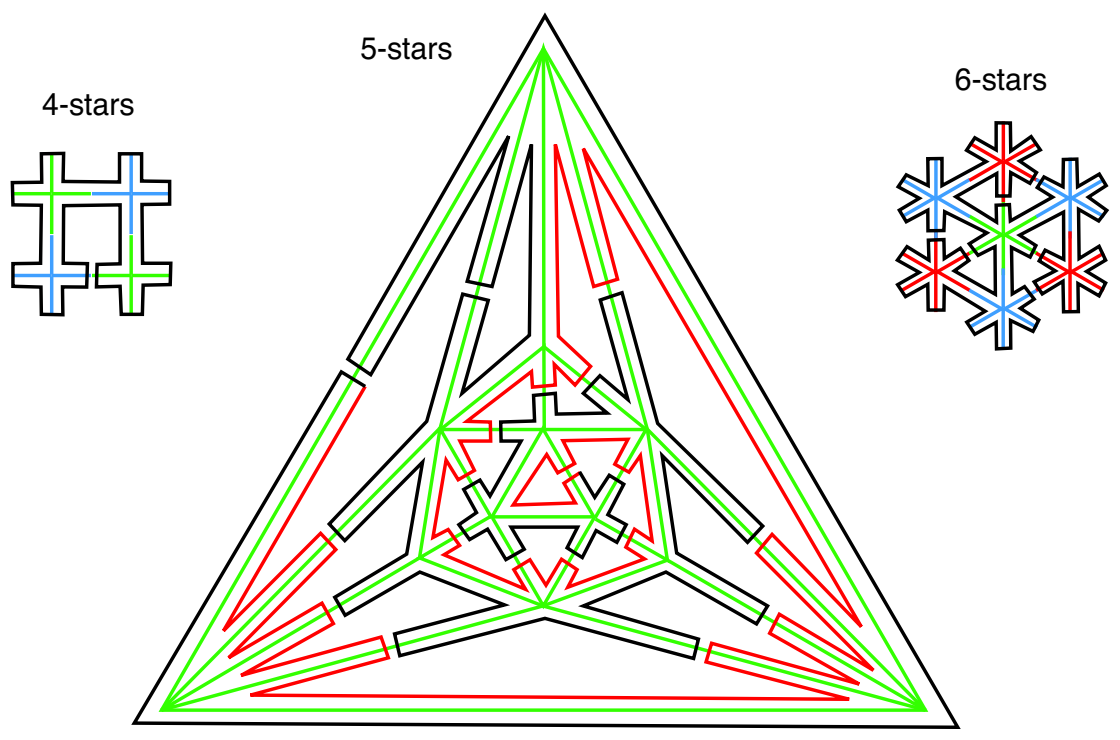

3,4,5,6-stars

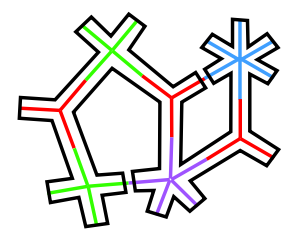

Fig. 11. Figures constructed using 4-stars, 5-stars and 6-stars.

large sheets with the interesting property that, although made from DNA "helices", no strands would cross from one surface of the sheet to the other!

Simply proposing a scheme for a general architecture, as this paper has done, isn't enough. A complete generalized approach would have three parts: (1) the definition of an infinite family of DNA shapes (2) the experimental demonstration of a convincing and representative set of examples and (3) the creation of automated design tools for the family of shapes. The last of these parts, while seeming a simple matter of software engineering, is of equal importance to the first two. It will allow the community of DNA nanotechnologists to reproduce and extend eachother's work but, of more importance perhaps, it will allow scientists outside of the community - physicists, chemists, materials scientists and biologists - to make and explore DNA nanostructures of their own design. As we create architectures and tools that put DNA nanotechnology into the hands of the research community at large, it will be exciting to see the legacy of Ned's flying DNA fish continue to grow. 


\section{Acknowledgements}

I thank Erik Winfree and William Shih for comments and the Caltech Center for the Physics of Information for a postdoctoral fellowship.

\section{References}

1. J. Chen and N.C. Seeman. The synthesis from DNA of a molecule with the connectivity of a cube. Nature, 350:631-633, 1991.

2. L.M. Adleman. Molecular computation of solutions to combinatorial problems. Science, 266:1021-1024, 1994.

3. E. Winfree. On the computational power of DNA annealing and ligation. In R.J. Lipton and E.B. Baum, editors, DNA Based Computers, volume 27 of DIMACS, pages 199-221, Providence, RI, 1996. AMS Press.

4. E. Winfree, F. Liu, L.A. Wenzler, and N.C. Seeman. Design and self-assembly of two-dimensional DNA crystals. Nature, 394:539-544, 1998.

5. C. Mao, T.H. LaBean, J.H. Reif, and N.C. Seeman. Logical computation using algorithmic self-assembly of DNA triple-crossover molecules. Nature, 407(6803):493-496, 2000.

6. P.W.K. Rothemund, N. Papadakis, and E. Winfree. Algorithmic self-assembly of DNA sierpinski triangles. PLoS Biology, 2(12):e424, 2004.

7. B. Yurke, A.J. Turberfield, A.P. Mills, Jr., F.C. Simmel, and J.L. Neumann. A DNA-fuelled molecular machine made of DNA. Nature, 406:605-608, 2000.

8. P. Yin, H. Yan, X.G. Daniell, A.J. Turberfield, and J.H. Reif. A unidirectional DNA walker that moves autonomously along a track. Angewandte Chemie International Edition, 43(37):4906-4911, 2004.

9. W.B. Sherman and N.C. Seeman. A precisely controlled DNA biped walking device. Nanoletters, 4(7):1203-1207, 2004.

10. J.S. Shin and N.A. Pierce. A synthetic DNA walker for molecular transport. Journal of the American Chemical Society, 126(35):10834-10835, 2004.

11. N.C. Seeman. Nucleic-acid junctions and lattices. Journal of Theoretical Biology, 99:237-247, 1982.

12. T.-J. Fu and N.C. Seeman. DNA double-crossover molecules. Biochemistry, 32:3211-3220, 1993

13. Paul W. K. Rothemund, Axel Ekani-Nkodo, Nick Papadakis, Ashish Kumar, D. Kuchnir Fygenson, and Erik Winfree. Design and characterization of programmable DNA nanotubes. Journal of the American Chemical Society, 26(50):16344-16353, 2004.

14. T.H. LaBean, H. Yan, J. Kopatsch, F. Liu, E. Winfree, J.H. Reif, and N.C. Seeman. Construction, analysis, ligation, and self-assembly of DNA triple crossover complexes. Journal of the American Chemical Society, 122:1848-1860, 2000.

15. H. Yan, X. Zhang, Z. Shen, and N.C. Seeman. A robust DNA mechanical device controlled by hybridization topology. Nature, 415:62-65, 2002.

16. W.M. Shih, J.D. Quispe, and G.F. Joyce. A 1.7-kilobase single-stranded DNA that folds into a nanoscale octahedron. Nature, 427(6453):618-621, 2004.

17. P.W.K. Rothemund. Generation of arbitrary nanoscale shapes and patterns by scaffolded DNA origami. submitted, 2005. 
18. Y. Zhang and N.C. Seeman. The construction of a DNA truncated octahedron. Journal of the American Chemical Society, 116:1661-1669, 1994.

19. N.C. Seeman. Construction of three-dimensional stick figures from branched DNA. DNA and Cell Biology, 7(10):475-486, 1991.

20. A.I.H. Murchie, R.M. Clegg, E. von Kitzing, D.R. Duckett, S. Diekmann, and D.M.J. Lilley. Fluorescence energy transfer shows that the four-way DNA junction is a right-handed cross of antiparallel molecules. Nature, 341:763-766, 1989.

21. D.R. Duckett, A.I.H. Murchie, S. Diekmann, E. von Kitzing, B. Kemper, and D.M.J. Lilley. The structure of the Holliday junction, and its resolution. Cell, 55:79-89, 1988.

22. C.D. Mao, W.Q. Sun, and N.C Seeman. Designed two-dimensional DNA holiday junction arrays visualized by atomic force microscopy. Journal of the American Chemical Society, 121:5437-5443, 1999.

23. P.W.K. Rothemund. DNA self-assembly with floppy motifs - single crossover lattices. Foundations of Nanoscience, Self-Assembled Architectures and Devices, pages 185-186, 2005.

24. H. Yan, S.H. Park, G.F., J.H. Reif, and T.H. LaBean. DNA-templated selfassembly of protein arrays and highly conductive nanowires. Science, 301:18821884,2003

25. Y. He, Y. Chen, H. Liu, A.E. Ribbe, and C. Mao. Self-assembly of hexagonal DNA two-dimensional (2D) arrays. Journal of the American Chemical Society, page 10.1021/ja0541938, 2005.

26. W.B. Sherman and N.C. Seeman. The design of nucleic acid nanotubes. Journal of Biomolecular Structure and Dynamics, 20(6):930-931, 2003.

27. Z.Y. Shen, H. Yan, T. Wang, and N.C. Seeman. Paranemic crossover DNA: A generalized Holliday structure with applications in nanotechnology. Journal of the American Chemical Society, 126:1666-1674, 2004. 



\section{Index}

Adleman, Leonard, 1 atomic force microscopy micrograph of a DNA "NED", 6

Chen, Junghuei, 1

DNA design methods comparison based on strand composition, 3-5

general architectures for, 15-17

multi-stranded, 3-5

scaffolded origami, 1-19

single-stranded origami, $3-5$

DNA motifs antiparallel crossover, 2-3

double-crossover, 2

paranemic crossover, 16

DNA nanotechnology, 1

future prospects, 15-17

principles, 3

DNA origami scaffolded, 1-19

for parallel multicrossovers, 2-7 for polygonal networks, $7-17$ single-stranded, 3-5, 16

geometrical DNA objects

buckminsterfullerene analog, 15

cube, 1,3

dodecahedron, 12, 14-16

icosahedron, 15-17

octahedron, 3

truncated octahedron, 6

LaBean, Thomas H., 7

Seeman, Nadrian C.

"NED" rendered in DNA, 6-7

his character, 1-2

Sherman, William, 15

Shih, William, 3, 16

Winfree, Erik, 1

Yan, Hao, 7 
